\title{
Sejamos Pragmáticos: Pesquisas em Sistemas de Informação com Relevância e Rigor
}

\section{Title: Let's be Pragmatic: Research in Information Systems with Relevance and Rigor}

\author{
Humberto C. C. Silva1, Alexandre O. Siqueira ${ }^{1}$, Marcus A. V. Araújo², Jairo S. \\ Dornelas $^{1}$ \\ ${ }^{1}$ Programa de Pós-Graduação em Administração \\ Universidade Federal de Pernambuco (UFPE) \\ Recife - PE - Brasil \\ ${ }^{2}$ Mestrado Profissional em Gestão Empresarial \\ Faculdade Boa Viagem (FBV | DeVry Brasil) \\ Recife - PE - Brasil \\ \{humberto.ccs, alex.pisciano\}@gmail.com, marcusaugusto77@hotmail.com, \\ jairo@ufpe.br
}

\begin{abstract}
Pragmatism can be defined as a doctrine that considers things from a practical point of view. Pragmatism preaches that action and practical relevance should be the key points of scientific research. The pragmatic paradigm has been presented as a paradigmatic alternative of positivism and interpretivism, enabling the solution of inherent problems of dominant paradigms, the positivism and interpretivism. The present article presents, from a bibliographical survey, the pragmatic paradigm, making a comparison with other paradigms, as well as the use of quantitative, qualitative and mixed methods, from a perspective of practical relevance with methodological rigor.
\end{abstract}

Keywords. Pragmatic paradigm; Research in Information Systems; Mixed methods; Rigor and relevance

Resumo. O pragmatismo pode ser definido como uma doutrina que considera as coisas a partir de um ponto de vista prático. O pragmatismo prega que a ação e a relevância prática devem ser os pontos chave da pesquisa científica. O paradigma pragmático tem se apresentado como alternativa paradigmática do positivismo e do interpretativismo, possibilitando a solução de problemas inerentes dos paradigmas dominantes, positivismo e interpretativismo. $O$ presente artigo apresenta, a partir de um levantamento bibliográfico, o paradigma pragmático, realizando uma comparação com outros paradigmas, assim como a utilização de métodos quantitativos, qualitativos e mistos, a partir de uma perspectiva de relevância prática com rigor metodológico.

Palavras-Chave. Paradigma pragmático; Pesquisa em Sistemas de Informação; Métodos mistos; Rigor e relevância

Cite as: Silva, H. C. C., Siqueira, A. O., Araújo, M. A. V., \& Dornelas, J. S. (2017). Sejamos Pragmáticos: Pesquisas em Sistemas de Informação com Relevância e Rigor (Let's be Pragmatic: Research in Information Systems with Relevance and Rigor). iSys: Revista Brasileira de Sistemas de Informação (Brazilian Journal of Information Systems), 10(4), 66-79. 


\section{Introdução}

Hevner e Chatterjee (2010) iniciam a discussão no livro "Design research in information systems: theory and practice" chamando a atenção para a necessidade de rigor e relevância prática da pesquisa em Sistemas de Informação (SI). Seguindo esta linha de relevância prática, é importante que um paradigma de pesquisa possa alinhar-se com estes dois requisitos, de rigor e relevância, sendo aqui sugerido o paradigma pragmático, conforme proposto por William James (1842-1910), John Dewey (18591952), Charles Sanders Peirce (1839-1914) e Herbert Mead (1863-1931) (PARVAIZ et $a l ., 2016)$, como opção aos paradigmas mais utilizados na condução de pesquisas em SI.

A busca pela relevância prática da pesquisa em SI não é assunto novo, já em 1999, Benbasat e Zmud (1999) questionaram os resultados práticos das pesquisas na área, abrindo seu artigo com um comentário de Scott Cowen, então reitor da Weatherhead School of Management da Case Western Reserve University, que $80 \%$ da pesquisa em gestão é irrelevante (BENBASAT; ZMUD, 1999). Neste tom de relevância prática da pesquisa, Baskerville e Myers (2004) afirmam que o pragmatismo, como filosofia de pesquisa, concentra-se em fazer as questões certas e em responder, empiricamente, à essas questões.

A abordagem de pesquisa que irá responder às questões levantadas em qualquer estudo deve seguir um posicionamento filosófico que irá afetar a forma do pesquisador ver o mundo e, assim, as escolhas que o mesmo irá fazer (PARVAIZ et al., 2016). Mackenzie e Knipe (2006) afirmam que vários são os posicionamentos paradigmáticos que podem ser adotados para a condução de uma pesquisa em SI, como positivista, construtivista, interpretativista, transformativo, emancipatório, crítico, desconstrutivista e pragmático. Entretanto, a discussão paradigmática está concentrada em dois polos, o positivista e o interpretativista (DARKE et al., 1998; GOLDKUHL, 2012; MORGAN, 2007).

Lee (1999), ao tratar do positivismo em ciências sociais, afirma que este é uma crença que a pesquisa em ciências sociais deveria emular como a pesquisa é feita em ciências naturais. As pesquisas em ciências naturais buscam a verdade em proposições formais, utilizando o método hipotético-dedutivo. O próprio Lee (1999) segue sugerindo que a pesquisa em SI deveria, na verdade, emular a pesquisa realizada em ciências aplicadas como medicina ou direito. As pesquisas em ciências aplicadas deveriam buscar a efetividade no agir.

No outro polo, o interpretativista, a busca é pelo entendimento do participante da situação que está sendo estudada. Assim, o pesquisador tende a utilizar, de forma mais comum, métodos de coleta de dados qualitativos, sendo os métodos quantitativos apenas suporte dos dados qualitativos (MACKENZIE; KNIPE, 2006). Orlikowski e Baroudi (1991) apontam que a filosofia de pesquisa interpretativista pode sofrer críticas, pois esta perspectiva não examina as condições que levaram a certos significados em algumas experiências relatadas, ou não explica as consequências não intencionais de uma ação, pois estas são forças significantes na construção da realidade social. Já uma abordagem pragmática, que tem como proposta a proximidade do fenômeno e a triangulação de fontes e métodos, possibilita formas de fugir dessa dependência de conceitos dos participantes da pesquisa (GOLDKUHL, 2004).

A discussão entre positivismo e interpretativismo vem sendo travada há algum tempo. Alguns estudos tentam fazer uma integração entre estas duas visões de mundo (LEE, 1991; WEBER, 2004), entretanto outros insistem que essas diferenças são 
grandes e irreconciliáveis (ORLIKOWSKI; BAROUDI, 1991). As irreconciliáveis diferenças entre as duas visões de mundo, positivista e interpretativista, ficam ainda mais evidentes quando tratamos de métodos de pesquisa mistos. Nestes casos, a objetividade e a subjetividade devem contribuir para a resposta da pergunta de pesquisa, e as visões antagônicas de mundo podem trazer problemas para o pesquisador. Assim, a perspectiva pragmática surge como uma alternativa viável, sendo esta a mais indicada na condução de pesquisas de caráter misto (MACKENZIE; KNIPE, 2006; MORGAN, 2007; PARVAIZ et al., 2016; FEILZER, 2010).

Ao utilizar a perspectiva pragmática o pesquisador se permite utilizar uma série de métodos como a pesquisa-ação (BASKERVILLE; MYERS, 2004), ou outros métodos de pesquisa qualitativa (GOLDKUHL, 2012; RATHBUN, 2008), utilizar apenas métodos quantitativos ou uma combinação dos dois (MACKENZIE; KNIPE, 2006). O foco está na solução do problema levantado, o que leva a uma abordagem "direcionada ao problema", próxima ao que é proposto por Davis (2010).

Assim, considerando o contexto de pesquisas na área de SI e a necessidade da realização de pesquisas com rigor e relevância prática, o presente estudo busca apresentar o paradigma pragmático como alternativa paradigmática ao positivismo e ao interpretativismo na realização de pesquisas em SI com rigor metodológico e foco na relevância prática do estudo.

Este trabalho, a partir de um levantamento bibliográfico (GALVÃO, 2010), contribui com a literatura sobre paradigmas de pesquisa e métodos de pesquisa em SI a partir da discussão sobre a utilização do paradigma pragmático para a realização de pesquisas em SI, no apelo para a realização de pesquisas que tenham relevância prática e na utilização de métodos mistos na condução de campo de pesquisas em SI.

O presente trabalho está estruturado em cinco sessões. Inicialmente será apresentado o paradigma pragmático. A segunda parte apresenta a relação do pragmatismo e a dicotomia entre as perspectivas positivista e interpretativista. Na terceira parte serão tratados os métodos de pesquisa quantitativo, qualitativo e misto, e como o paradigma pragmático possibilita sua conciliação. A quarta seção trará a discussão de rigor e relevância. E, finalmente, na quinta parte, serão apresentadas as considerações finais do estudo.

\section{O Pragmatismo}

O pragmatismo, na literatura, é tratado como paradigma de pesquisa (GOLDKUHL, 2012), como uma abordagem metodológica (PARVAIZ et al., 2016), ou como um posicionamento filosófico (GOLDKUHL, 2004). Sua origem foi o chamado pragmatismo americano, presente nos trabalhos de Peirce (1931), James (1907), Dewey (1931) e Mead (1938). Apesar de seu berço americano, o pragmatismo encontra ressonância em pensadores europeus como Arens (1994) e Thayer (1981), e na filosofia asiática como descrito por Shusterman (2004).

De acordo com Dewey (1931), um empiricismo que se contenta em repetir fatos já conhecidos não tem lugar para possibilidade nem para a libertação. Assim, o pragmatismo tem interesse não só no que "é”, mas no que "deveria ser". Essa maneira de perceber o mundo faz com que o pragmático busque a realização de mudanças em direções desejadas e a ação de mudança, que é um interesse central do pragmatismo, deve ser guiada por propósito e conhecimento. Dessa forma, o mundo seria transformado através da razão e da ação, que estão ligados de forma inseparável com o 
conhecimento humano e a ação humana (GOLDKUHL, 2004).

A palavra 'Pragma', de origem grega " $\pi \rho \alpha ́ \gamma \mu \alpha$ ", significa ação, e o termo 'pragmático' tem a conotação de busca por soluções possíveis e executáveis para complexos problemas humanos. Assim, Kinouchi (2007) define pragmatismo como uma doutrina que considera as coisas a partir de um ponto de vista prático. Já Mackenzie e Knipe (2006) afirmam que o pragmatismo não está comprometido com nenhum sistema filosófico, e que os pesquisadores pragmáticos focam no "que" e "como" do problema de pesquisa.

A partir dos conceitos de Peirce, James, Dewey e Mead, surgem premissas que fundamentam a filosofia pragmática (BASKERVILLE; MYERS, 2004). A primeira é baseada no princípio de Peirce de que todos os conceitos humanos são definidos a partir das suas consequências. Uma concepção intelectual, ou uma ideia, deve considerar suas consequências práticas, sendo a soma dessas consequências o significado da concepção ou da ideia (PEIRCE, 1905).

Seguindo as premissas fundamentais da filosofia pragmática, o princípio de James, segunda premissa, trata da verdade, afirmando que a verdade está personificada no resultado prático da ação. Para James (1907) a maioria dos nossos pensamentos é usado para mudar o mundo, sendo, assim, necessário saber o que temos que mudar. Portanto, a mente engendra a verdade na realidade, pois sabemos o que temos que mudar, e a verdade teórica, que para James (1907) é esse conhecimento da realidade, deve vir antes da aplicação prática.

A terceira premissa, baseada na lógica da investigação controlada de Dewey (1931), na qual o pensamento racional é intercalado com a ação. Para Dewey (1931) o pragmatismo é a teoria da investigação, no qual as ideias tomam formas lógicas em um processo chamado de investigação controlada, que é ao mesmo tempo rigoroso cientificamente e senso comum diário. A investigação é, então, a transformação direta ou controlada de uma situação indeterminada em outra que é determinada em suas distinções e relações, de forma que os elementos da situação original são convertidos em um novo todo unificado.

Baskerville e Myers (2004), ao tratar da lógica de Dewey, apresentam cinco elementos característicos que são comuns a todas as formas de investigação humana: (1) uma situação indeterminada; (2) a formulação de um problema; (3) a determinação de uma solução; (4) o raciocínio ou o pensamento aprofundado das aplicações das soluções encontradas; e (5) a operacionalização das soluções em fatos ou ações.

Finalmente, na quarta premissa, Mead (1938) propõe que a ação humana é contextualizada socialmente e que a conceituação humana é, também, um reflexo social destas ações. Como consequência da perspectiva pragmática, Mead (1938) percebe que a interação humana molda a ação humana, e como a ação leva a consequências práticas, então a configuração social molda conceitos como verdade, racionalidade e, até mesmo, o conceito de ação prática em si.

Nesta linha da ação e da aplicação prática, Dewey (1931) esclarece que o pragmatismo não se preocupa com fenômenos antecedentes, mas com fenômeno consequentes e sobre as possibilidades da ação. Ao utilizar o ponto de vista pragmático é possível perceber que ideias gerais tem um diferente papel a desempenhar que o de apenas reportar e registrar experiências passadas, elas são as bases para organizar futuras observações, experiências e aplicações. 
Feilzer (2010) acrescenta que o pragmatismo deixa de lado questões como verdade e realidade, aceitando, filosoficamente, que existem realidades singulares e múltiplas, que estão abertas a questionamento empírico e que devem ser orientadas para a resolução de problemas existentes no "mundo real". Assim, o pesquisador pragmático estaria livre de barreiras mentais e práticas impostas pela dicotomia positivismo $\mathrm{x}$ construtivismo, e não seria prisioneiro de uma metodologia ou técnica de pesquisa particular (CRESWELL; CLARK, 2007; ROBSON; MCCARTAN, 2016).

Então, para Goldkuhl (2004), não é suficiente apenas observar e então generalizar. O conhecimento não pode ser apenas um sumário de experiências passadas, mas que a razão tem uma função criativa que ajuda na criação do mundo, de forma que este está em transformação. Esta transformação é constante e presente na prática de SI, e como o interesse do pragmático está na mudança e na ação, é um dever moral dar prioridade às mudanças que tenham um caráter positivo à sociedade. Isto é, o valor da pesquisa de SI para a sociedade está associado às possibilidades de melhorias nas práticas de SI (GOLDKUHL, 2004).

Adicionalmente, Goldkuhl (2008) afirma que três tipos diferentes de pragmatismo podem ser reconhecidos. São eles o pragmatismo funcional, pragmatismo referencial e o pragmatismo metodológico. Estes três tipos de pragmatismos estão relacionados à três questões fundamentais:

- Por que conhecer?

- O que conhecer?

- Como conhecer?

O pragmatismo funcional, que tem como princípio a descoberta para a ação, ou seja, a ação é o propósito. Funcional significa que o conhecimento deveria ser útil e aplicável, ou seja, o conhecimento que tem uma característica prescritiva, como modelos e métodos, é importante no pragmatismo funcional. Além da abordagem sistemática e tradicional da criação de hipóteses (CRONEN, 2001), no pragmatismo funcional é possível a utilização de Design Science e Design Theories (HEVNER et al., 2004);

O pragmatismo referencial, que utiliza o conhecimento sobre a ação, no qual a ação é o objeto. Neste tipo de pragmatismo, a preocupação é descrever o mundo de uma forma orientada à ação. Várias são as abordagens teóricas que influenciam a pesquisa em SI como teoria da estruturação de Giddens, teoria da ação comunicativa de Habermas, teoria da ação (ENGESTRÖM, 1987) e teoria da prática (SCHATZKI et al, 2001). Em termos metodológicos, Goldkuhl (2008) cita a Action Workflow e a metodologia DEMO (Dynamic Essential Modeling of Organizations) (DIETZ, 1999).

Finalmente, o pragmatismo metodológico, no qual o conhecimento é obtido através da ação, ou seja, a ação é a origem e o meio para a descoberta. O desenvolvimento do conhecimento é baseado em interações contínuas entre conhecer e agir. A experimentação e exploração são centrais no processo de descoberta. Já a pesquisa-ação o método de preferência do pragmatismo metodológico (GOLDKUHL, 2008).

\section{O Pragmatismo e a Dicotomia Positivista $x$ Interpretativista}

Segundo Feilzer (2010), um paradigma pode ser considerado um padrão ou modelo 
aceito, uma estrutura organizada, ou, ainda, um posicionamento filosófico profundo em relação à natureza do fenômeno social e das estruturas sociais. Um paradigma se reafirma ao excluir outros, e articular teorias a partir da sua visão (KUHN, 1962). Assim, entre a objetividade e captura da realidade do positivismo, e a subjetividade plural do interpretativismo, os paradigmas podem ser interpretados como prescritivos, requisitando de métodos de pesquisa específicos, e excluindo outros. Dessa forma, o paradigma pode restringir a curiosidade e criatividade e, até mesmo, cegar pesquisadores de aspectos chave do fenômeno social (FELIZER, 2010).

Essa possível "cegueira" do pesquisador social pode ser percebida quando Mertens (2014), ao definir o uso do positivismo nas ciências sociais, afirma que o positivismo pode ser aplicado ao mundo social a partir da suposição de que o mundo social pode ser estudado da mesma maneira que o mundo natural. Nesta linha, O'Leary (2017) acrescenta que o positivismo objetiva ao teste de teorias ou a descrição de experiências, a partir da observação e da medição, para que seja possível predizer e controlar as forças que nos cercam. Essa visão dura da realidade pura, capturável e previsível, contrasta, até certo ponto, com aquela visão que é anunciada pelos póspositivistas. Para estes o mundo é ambíguo, variável e possuidor de múltiplas realidades. Assim, o que é verdade para uma pessoa ou um grupo, pode não ser para outros (O'LEARY, 2017). Entretanto, pesquisadores positivistas e pós-positivistas estão comumente alinhados com métodos de coleta e análise quantitativos (MACKENZIE; KNIPE, 2006).

Morgan (2007) lembra que essa preferência sobre a abordagem quantitativa, e as claras limitações que esta visão tem, poderia ter gerado uma discussão em relação às possibilidades que a abordagem qualitativa traria ao meio científico. Entretanto as discussões se concentraram mais em questões filosóficas, baseadas em conceitos de filosofia do conhecimento. $\mathrm{O}$ questionamento sobre anomalias encontradas no paradigma dominante, um dos pressupostos de Kuhn (1962) para a mudança paradigmática, levou a uma tentativa de criação de um paradigma alternativo, chamado por Morgan (2007) de paradigma metafísico e por Guba e Lincoln (1988) de paradigma construtivista.

Em relação aos métodos qualitativos, uma importante discussão é se a pesquisa qualitativa é igual, ou está sempre relacionada, ao interpretativismo (GOLDKUHL, 2012). Para Trauth (2001) o interpretativismo é a lente que mais influencia a utilização de métodos qualitativos. Entretanto, para Myers e Avison (2002), a expressão "pesquisa qualitativa" não é sinônimo de interpretativismo, e que as pesquisas qualitativas podem ser positivistas, críticas ou interpretativistas, mas que essa escolha está ligada à pressupostos filosóficos do pesquisador. Apesar das diferentes possibilidades de paradigmas relacionados à pesquisa qualitativa, Morgan (2007) lembra que grande parte do debate paradigmático existente está relacionado à rivalidade entre o interpretativismo e o positivismo, e uma terceira via, assim como proposto por Wicks e Freeman (1998) e Fishman (1999), seria o paradigma pragmático. Uma proposição semelhante foi feita por Goles e Hirschheim (2000) para pesquisas em SI, na qual o paradigma pragmático deveria ser incluído no debate de possibilidades paradigmáticas.

A alternativa pragmática nos mostra como é possível resolver problemas relacionados ao paradigma positivista, como visão dura da realidade e a possibilidade exata de medição e prescrição, como também problemas relacionados ao paradigma interpretativista, como a dificuldade na determinação de fronteiras, os problemas 
relacionados à incomensurabilidade e as relações entre o pensamento paradigmático e as práticas de pesquisa (MORGAN, 2007).

Apesar das fundações do pensamento pragmático não serem completamente conhecidas e reconhecidas, a sua influência em pesquisas de SI é extensa e presente no dia a dia de pesquisa. Baskerville e Myers (2004) argumentam sobre a utilização do pragmatismo como fundamentação pragmática na condução de pesquisas ação, assim como Lee e Nickerson (2010) afirmam que o pragmatismo é o paradigma de pesquisa mais apropriado na condução de Research Design do que o positivismo. Como o pragmatismo está preocupado com a ação e mudança e com a interação entre o conhecimento e a ação, este seria o paradigma mais apropriado em casos em que a intervenção é uma mudança organizacional ou a construção de artefatos (GOLDKUHL, 2012).

Morgan (2007) nos apresenta, de forma resumida, como as metodologias qualitativa, quantitativa e pragmática, abordam as relações entre teoria e dados, o processo de pesquisa e a inferência a partir dos dados. As proposições de Morgan (2007) estão na Tabela 1.

Tabela 1: Questões-chave na Metodologia de Pesquisa em Ciências Sociais. Fonte: Morgan (2007)

\begin{tabular}{llll}
\hline & $\begin{array}{l}\text { Abordagem } \\
\text { Qualitativa }\end{array}$ & $\begin{array}{l}\text { Abordagem } \\
\text { Quantitativa }\end{array}$ & $\begin{array}{l}\text { Abordagem } \\
\text { Pragmática }\end{array}$ \\
\hline Conexão entre teoria e dados & Indução & Dedução & Abdução \\
Relação com o processo de pesquisa & Subjetividade & Objetividade & Intersubjetividade \\
Inferência a partir dos dados & Contextual & Generalização & Transferência \\
\hline
\end{tabular}

\section{Pesquisas Quantitativas, Qualitativas e Métodos Mistos}

A matriz de conexão da teoria de Laughlin (1995) é uma das ferramentas que podem ser utilizadas para a escolha de uma abordagem metodológica apropriada para a pesquisa. De acordo com essa matriz, todo processo de pesquisa é composto por três grandes dimensões que são a teoria, a metodologia e a mudança. Dessa forma, a escolha do pesquisador por um paradigma de pesquisa passará pela aplicação em maior ou menor grau de cada uma dessas dimensões.

Assim, no paradigma positivista o objetivo é a utilização da dedução para a confirmação de teorias bem estabelecidas, utilizando-se da análise de dados a partir de uma abordagem quantitativa. Já no interpretativismo, a abordagem indutiva objetiva a descoberta de uma teoria ou um aprofundamento da compreensão sobre um fenômeno (PARVAIZ et al. 2016). Portanto, enquanto o positivismo enfatiza as leis que regem uma realidade bruta e independente de observação e o interpretativismo enfatiza o papel da criatividade, da subjetividade e da realidade individual, o pragmatismo rejeita ambos, pois nenhuma teoria pode ser totalmente objetiva, falseável, etc., como propõe o positivismo, nem totalmente maleável e interpretativamente satisfazer a qualquer situação, como possivelmente ocorre com os resultados subjetivos propostos pelo interpretativismo (POWELL, 2001).

A questão da dicotomia positivismo $\mathrm{x}$ interpretativismo em pesquisas em ciências humanas, e também em pesquisas em SI, é discutida na academia (GOLDKUHL, 2012; LEE, 1991; WEBER, 2004), entretanto um ponto que Orlikowski 
e Baroudi (1991) levantam é a impossibilidade de conciliação entre esses dois paradigmas. Esta separação, inconciliável, entre os dois paradigmas, positivista e interpretativista, reflete-se em questões metodológicas, nas quais quantitativistas e qualitativistas se veem em extremos opostos incapazes de ver "além da sua colina metodológica".

Já para os pragmáticos, o mundo real existe, mas, ao mesmo tempo, cada indivíduo tem sua interpretação única desse mundo (MORGAN, 2007). Assim, o pragmático não aceita ou confia apenas na subjetividade, mas adota a noção de intersubjetividade, que permite aos pesquisadores capturar a dualidade, subjetiva e objetiva, de um fenômeno antes de representá-lo como uma realidade social (PARVAIZ et al., 2016).

Se pensarmos em uma reta representando os dois paradigmas de pesquisa, positivista e interpretativista, no qual de um lado temos o positivismo/pós-positivismo e do outro temos o interpretativismo, o pragmatismo surgiria como o paradigma que poderia agregar os dois extremos. Os métodos quantitativos, típicos dos estudos positivistas, e os métodos qualitativos, igualmente presentes nos estudos interpretativistas, são utilizados sem restrição em estudos de cunho pragmático (MACKENZIE; KNIPE, 2006; PARVAIZ et al., 2016), assim como a junção destes, quantitativo e qualitativo, na realização de estudos com métodos mistos (TEDDLIE; TASHAKKORI, 2003).

Tashakkori e Teddlie (2003) são autores que apontam a importância da utilização de métodos mistos. Estes métodos mistos envolvem um desenho de pesquisa no qual a operacionalização passa pela utilização de mais de um método de pesquisa e, assim, mais de uma visão de mundo, quantitativa e qualitativa. Para Creswell (2013), a técnica de métodos mistos é aquela em que o pesquisador baseia suas alegações em elementos pragmáticos. Assim, Tashakkori e Teddlie (2003) propõem que o pragmatismo, ou a "filosofia da livre escolha" (SYMONDS; GORARD, 2008, p. 3), é a epistemologia mais apropriada para a utilização de métodos mistos. Os proponentes da utilização de pesquisas que utilizam métodos mistos apreciam o valor que pode ser obtido a partir da visão de mundo quantitativa e qualitativa, permitindo o melhor entendimento do fenômeno estudado (VENKATESH; BROWN; BALA, 2013).

Nos estudos em SI, os métodos qualitativos têm sido usados, tipicamente, para estudos exploratórios de modo a desenvolver um entendimento mais aprofundado de um fenômeno, ou para a geração de novos insights teóricos (WALSHAM, 2006), enquanto os métodos quantitativos são usados para estudos confirmatórios, de maneira a testar teorias (VENKATESH; BROWN; BALA, 2013). Entretanto, para Teddlie e Tashakkori (2003), os métodos de pesquisa mistos tem a habilidade de responderem a questões de pesquisa no nível exploratório e confirmatório simultaneamente. Outra característica dos métodos mistos apontada por Teddlie e Tashakkori (2003) é a habilidade de prover inferências mais consistentes do que aquelas obtidas a partir de métodos ou visões de mundo singulares.

A área de estudos que o pesquisador de SI atua é de constante mudança, o que torna o meio extremamente complexo e nos apresenta novos desafios na busca pelo entendimento das capacidades, uso e impacto dessas novas tecnologias. Assim, é comum termos situações nas quais as proposições teóricas existentes não explicam o fenômeno. Portanto, a utilização de métodos mistos fornece ferramentas que auxiliam o pesquisador de SI na constante busca por melhores contribuições para a área, teóricas e 
práticas.

\section{Rigor e Relevância}

A questão sobre rigor e relevância vem sendo tratada na academia há alguns anos, com vários pesquisadores apontando a perda da relevância prática das pesquisas organizacionais e questionando sobre o futuro do campo (SCHULTZ, 2010). Já em 1999, Benbasat e Zmud alertaram para a falta de relevância prática da pesquisa em SI, questionando se o conhecimento produzido poderia ser aplicado por profissionais no seu dia a dia (BENBASAT; ZMUD, 1999).

A pesquisa em ciências sociais, e em particular na área de SI, que tenha a pretensão de ser relevante ao praticante, deve sempre responder a perguntas como "para que é isso?", "para quem é isso?" e "como realizar a pesquisa?". Esses três questionamentos nos mostram uma preocupação de aplicação prática, juntamente com a necessidade de realizar a pesquisa dentro de aspectos mínimos de rigor metodológico. Essas perguntas também trazem aspectos epistemológicos à construção da pesquisa, pois não existe a preocupação na reprodução exata da realidade, mas de uma solução para um problema, que alguém tem, a partir de uma pesquisa científica (FEILZER, 2009).

Lee (1999) argumenta que a pesquisa em SI deveria ser realiza da mesma maneira que em áreas profissionais como medicina ou direito e não utilizando conceitos herdados das ciências naturais. Mais recentemente Baskerville e Myers (2004) realizaram uma chamada de trabalhos para pesquisas com mais relevância prática, com pesquisas que utilizassem como método a pesquisa-ação.

De acordo com Schultz (2010), a pesquisa em ciências sociais persegue um tipo de rigor científico que não foi desenvolvido internamente, mas utilizando-se de metodologias importadas, ou imitadas, das ciências naturais. É bem verdade que foi construída uma vertente em ciências sociais na qual a especificidade do ser humano passou a ser tema central, resultando no paradigma interpretativista (SANTOS,1999). Entretanto, quando falamos de pesquisas em várias áreas das ciências sociais como marketing (DE ANDRADE et al., 2017), finanças (PINTO et al., 2016) e até mesmo sistemas de informações (KWAN, 2017), o campo ainda se mostra positivista.

Assim, as requisições de elevada padronização de argumentação, teorização e apresentação de dados empíricos, solicitadas por várias revistas "forçam" as organizações a serem instituições de lógica dura nos estudos organizacionais. Quando essa lógica forçada funciona, novas teorizações e conceitos são criados, mas a falha leva a situações nas quais as organizações tem que se adaptar ao "fenômeno aceitável cientificamente" (SCHULTZ, 2010).

O desafio de tentar entender o mundo organizacional a partir da visão das ciências naturais pode ser percebido nas palavras de Lee (1999, p. 30) quando ele coloca que "o mundo não para enquanto as ciências naturais desenvolvem o conhecimento que ajude a resolver problemas do mundo real". O conhecimento do mundo real é produzido independentemente de qualquer ciência natural (LEE, 1999). Schultz (2010) alerta que o desbalanceamento entre o rigor e a prática leva a estudos excruciantemente detalhados, de problemas menores, que tem pouca generalização, e são aplicáveis em comunidades escolares extremamente especializadas.

Um dos pontos importantes do paradigma pragmático é o balanceamento entre 
rigor e relevância (SCHULTZ, 2010). Segundo Ulrich (2007), esse balanço entre relevância e rigor, tende a beneficiar a relevância, pois aí encontra-se o fundamento do pensamento pragmático. Entretanto, Ulrich (2007) segue afirmando que certos cuidados devem ser tomados para que essa balança seja equilibrada, e que o rigor metodológico seja, também, parte do estudo científico. Para Ulrich (2007) o embasamento ético cuidadoso é um ponto central, pois, sem esse embasamento ético o pensamento pragmático corre o risco de ser apenas um mero oportunismo ou um utilitarismo.

Especificamente em pesquisas na área de SI, Benbasat e Zmud (1999) trazem algumas sugestões de abordagens que devem ser evitadas quando são produzidos artigos científicos de forma que estes tenham maior aceitação na prática de gestores de SI. A primeira sugestão é evitar excessivo rigor em detrimento da relevância. A relevância prática, o objetivo de resolver problemas do dia a dia do praticante da gestão deve ser sempre o foco da produção científica. O rigor científico não pode ser esquecido, mas deve existir um equilíbrio entre rigor e relevância. Em seguida Benbasat e Zmud (1999) sugerem que falta, no campo, continuidade dos trabalhos. As pesquisas em SI tendem a apresentar um resultado e, então, serem abandonadas. É importante que exista continuidade e que novos achados complementem a teoria, formando uma tradição teórica.

Finalmente, o próprio objeto de estudo, os sistemas de informação e/ou a tecnologia de informação, são de altíssima dinamicidade. As mudanças e avanços tecnológicos fazem com que o estudo da utilização da tecnologia e seus impactos na sociedade torne-se uma corrida contra o tempo, pois é possível que um resultado de alta relevância prática, não tenha qualquer impacto um ano depois de sua publicação (BENBASAT; ZMUD, 1999).

\section{Conclusão}

A realização de uma pesquisa científica tem como premissa a escolha de uma visão epistemológica que irá guiar o pesquisador nas suas escolhas. Dentre os paradigmas dominantes na pesquisa em ciências sociais temos o positivista e o interpretativista. Estes, para Orlikowski e Baroudi (1991) são extremos opostos no que diz respeito ao pensamento filosófico e às escolhas metodológicas. Estas visões, tão diferentes de mundo, podem prejudicar a percepção dos fenômenos estudados ou, até mesmo, "cegar" o pesquisador para aspectos importantes da pesquisa. Assim, a perspectiva pragmática, ou paradigma pragmático, apresenta-se como alternativa viável para a constante discussão existente entre positivismo e interpretativismo.

O pragmatismo prega a busca do conhecimento a partir da ação como afirma Goldkuhl (2004, p. 24) "O pragmatismo significa o reconhecimento da dialética completa entre conhecimento e ação: a ação adequada é uma ação com conhecimento; o conhecimento adequado é o conhecimento ativo". Assim, utilizando-se uma afirmação de Lee (1999), a pesquisa em SI deveria ser uma pesquisa voltada à prática, voltada à solução de problemas vivenciados no dia a dia dos gestores de SI.

Outro ponto importante apresentado no presente artigo é que a escolha pelo paradigma pragmático não limita suas escolhas metodológicas. É verdadeira a afirmação de que é possível a realização de pesquisas qualitativas sob uma visão positivista e, de forma oposta, a realização de pesquisas quantitativas sob a ótica interpretativista. Entretanto, a posição filosófica de cada extremo, subjetivo vs objetivo, contextual vs generalista, tornam o cruzamento de métodos complicado, para dizer o 
mínimo. Já o pesquisador pragmático, tem a flexibilidade para percorrer os dois ambientes, quantitativo e qualitativo, e, até mesmo, juntá-los, quando o problema de pesquisa necessitar de vários níveis de pesquisa.

Finalmente, um ponto que deve ser colocado é o do rigor metodológico. As pesquisas de cunho pragmático têm como foco principal a relevância prática da pesquisa. Entretanto não se pode deixar de lado o rigor da pesquisa científica, sob pena de termos, ao final, um trabalho sem fundamentação, que pode até resolver um problema, mas, nas palavras de Ulrich $(2007$, p. 1110) "seria difícil evitar a suspeita de ser um mero oportunismo e utilitarismo".

Dificilmente um pesquisador muda sua escolha paradigmática, ainda mais quando essa escolha está relacionada a questões de formação pessoal e profissional. Quando falamos de escolhas paradigmáticas positivistas ou interpretativistas, o distanciamento torna-se palpável, para não dizer conflitante. Entretanto, a adoção de um paradigma pragmático possibilita um melhor ajuste menos traumatizante aos dois extremos paradigmáticos, permitindo que pesquisadores, sem entrar em grandes conflitos filosóficos, tenham acesso a outras ferramentas metodológicas que possibilitem explicar melhor o fenômeno estudado. Adicionalmente, é possível uma conciliação entre o pensamento positivista e o pragmático, assim como entre $o$ pensamento interpretativista e o pragmático, fazendo com que o pesquisador possa trafegar melhor, ou com um grau de esforço que não negue sua bagagem filosófica, entre paradigmas.

\section{Referências}

ARENS E. The logic of pragmatic thinking. From Peirce to Habermas, Humanities Press, Atlantic Highlands. 1994.

BASKERVILLE, Richard; MYERS, Michael D. Special issue on action research in information systems: Making IS research relevant to practice: Foreword. Mis Quarterly, p. 329-335, 2004. [GS Search]

BENBASAT, Izak; ZMUD, Robert W. Empirical research in information systems: the practice of relevance. MIS quarterly, p. 3-16, 1999. [GS Search]

CRESWELL, John W. Research design: Qualitative, quantitative, and mixed methods approaches. Sage publications, 2013.

CRESWELL, John W.; CLARK, Vicki L. Plano. Designing and conducting mixed methods research. Sage Publications. 2007.

CRONEN; V. Practical theory, practical art, and the pragmatic-systemic account of inquiry. Communication theory, Vol 11 (1), p 14-35, 2001. [GS Search]

DARKE, Peta; SHANKS, Graeme; BROADBENT, Marianne. Successfully completing case study research: combining rigour, relevance and pragmatism. Information systems journal, v. 8, n. 4, p. 273-289, 1998. [GS Search]

DAVIS, Gerald F. Do theories of organizations progress?. Organizational Research Methods, v. 13, n. 4, p. 690-709, 2010. [GS Search]

DE ANDRADE, Matheus Lemos et al. A Miopia Paradigmática e os Jogos da Verdade nas Pesquisas de Marketing. REMark, v. 16, n. 3, p. 383, 2017. [GS Search]

DEWEY, J. The development of American pragmatism. In H. S. Thayer (Ed.) (1989), 
Pragmatism: The classic writings (pp. 23-40). Indianapolis, IN: Hackett. 1931.

DIETZ, Jan LG. Understanding and modelling business processes with DEMO. In: ER. 1999. p. 188-202. [GS Search]

FEILZER, Martina Yvonne. Doing mixed methods research pragmatically: Implications for the rediscovery of pragmatism as a research paradigm. Journal of mixed methods research, v. 4, n. 1, p. 6-16, 2010. [GS Search]

FISHMAN, Daniel. The case for pragmatic psychology. NYU Press, 1999.

GALVÃO, Maria Cristiane Barbosa. O levantamento bibliográfico e a pesquisa científica. Fundamentos de epidemiologia. 2ed. A, v. 398, p. 1-377, 2010. [GS Search]

GOLDKUHL, Göran. Meanings of pragmatism: Ways to conduct information systems research. Action in Language, Organisations and Information Systems, 2004. [GS Search]

GOLDKUHL, Göran. Pragmatism vs interpretivism in qualitative information systems research. European Journal of Information Systems, v. 21, n. 2, p. 135-146, 2012. [GS Search]

GOLDKUHL, Göran. What kind of pragmatism in information systems research. In: AIS SIG Prag inaugural meeting. 2008. [GS Search]

GOLES, Tim; HIRSCHHEIM, Rudy. The paradigm is dead, the paradigm is dead... long live the paradigm: the legacy of Burrell and Morgan. Omega, v. 28, n. 3, p. 249268, 2000. [GS Search]

GUBA, Egon G.; LINCOLN, Yvonna S. Do inquiry paradigms imply inquiry methodologies. Qualitative approaches to evaluation in education, p. 89-115, 1988.

HEVNER, Alan; CHATTERJEE, Samir. Design research in information systems: theory and practice. Springer Science \& Business Media, 2010.

HEVNER, A. R.; MARCH, S.T.; PARK, J. Design Science in Information Systems Research. MIS Quaterly, v. 28, n. 1, p. 75-105, 2004. [GS Search]

JAMES, William. Pragmatism, a New Name for Some Old Ways of Thinking, Popular Lectures. 1907.

KUHN, Thomas S. The Structure of Scientific Revolutions Vol. 1962.

KWAN, Becky SC. A cross-paradigm macro-structure analysis of research articles in Information Systems. English for Specific Purposes, v. 45, p. 14-30, 2017. [GS Search]

LAUGHLIN, Richard. Empirical research in accounting: alternative approaches and a case for "middle-range" thinking. Accounting, Auditing \& Accountability Journal, v. 8, n. 1, p. 63-87, 1995. [GS Search]

LEE, Allen S. Rigor and relevance in MIS research: Beyond the approach of positivism alone. MIS quarterly, p. 29-33, 1999. [GS Search]

MACKENZIE, Noella; KNIPE, Sally. Research dilemmas: Paradigms, methods and methodology. Issues in educational research, v. 16, n. 2, p. 193-205, 2006. [GS Search] 
MEAD, George Herbert. The philosophy of the act. 1938.

MERTENS, Donna M. Research and evaluation in education and psychology: Integrating diversity with quantitative, qualitative, and mixed methods. Sage publications, 2014.

MORGAN, David L. Paradigms lost and pragmatism regained: Methodological implications of combining qualitative and quantitative methods. Journal of mixed methods research, v. 1, n. 1, p. 48-76, 2007. [GS Search]

MYERS, Michael D.; AVISON, David. An introduction to qualitative research in information systems. 2002.

O'LEARY, Zina. The essential guide to doing your research project. Sage, 2017.

ORLIKOWSKI, Wanda J.; BAROUDI, Jack J. Studying information technology in organizations: Research approaches and assumptions. Information systems research, v. 2, n. 1, p. 1-28, 1991. [GS Search]

PARVAIZ, Gohar Saleem et al. Pragmatism for Mixed Method Research at Higher Education Level. Business \& Economic Review, v. 8, n. 2, p. 67-79, 2016. [GS Search]

PEIRCE, Charles S. Collected Papers (Vols. 1-8). 1931.

PINTO, Nelson Guilherme Machado et al. A Aproximação De Trabalhos Acadêmicos No Universo Organizacional Nas Ciências Administrativas: Uma Análise Dos Trabalhos Do Encontro Da Associação Nacional De Pós-Graduação E Pesquisa Em Administração De 2014. Revista UNEMAT de Contabilidade, v. 5, n. 9, 2016. [GS $\underline{\text { Search] }}$

POWELL, Thomas C. Competitive advantage: logical and philosophical considerations. Strategic management journal, v. 22, n. 9, p. 875-888, 2001. [GS Search]

RATHBUN, Brian Christopher. Interviewing and qualitative field methods: pragmatism and practicalities. In: The Oxford handbook of political methodology. 2008.

ROBSON, Colin; MCCARTAN, Kieran. Real world research. John Wiley \& Sons, 2016.

SANTOS, Boaventura de Sousa. Um discurso sobre as ciências. Porto (Portugal): Afrontamento, 1999. [GS Search]

SCHULTZ, Majken. Reconciling pragmatism and scientific rigor. Journal of Management Inquiry, v. 19, n. 3, p. 274-277, 2010. [GS Search]

SHUSTERMAN, Richard. Pragmatism and East-Asian Thought. Metaphilosophy, v. 35, n. 1-2, p. 13-43, 2004. [GS Search]

SYMONDS, Jenny E.; GORARD, S. The death of mixed methods: research labels and their casualties. In: British Educational Research Association Annual Conference. 2008. [GS Search]

TASHAKKORI, Abbas; TEDDLIE, Charles. The Past and the Future of Mixed Methods Research: From 'Methodological Triangulation' to 'Mixed Methods Designs'. Handbook of mixed methods in social and behavioral research, p. 671-701, 2003.

TEDDLIE, Charles; TASHAKKORI, Abbas. Major issues and controversies in the use of mixed methods in the social and behavioral sciences. Handbook of mixed methods 
in social \& behavioral research, p. 3-50, 2003.

THAYER, Horace Standish. Meaning and action: A critical history of pragmatism. Hackett Publishing, 1981.

TRAUTH, Eileen M. The choice of qualitative methods in IS research. Qualitative Research in IS: Issues and Trends, 2001.

ULRICH, Werner. Philosophy for professionals: Towards critical pragmatism. Journal of the Operational Research Society, v. 58, n. 8, p. 1109-1113, 2007. [GS Search]

VENKATESH, Viswanath; BROWN, Susan A.; BALA, Hillol. Bridging the qualitativequantitative divide: Guidelines for conducting mixed methods research in information systems. MIS quarterly, v. 37, n. 1, 2013. [GS Search]

WALSHAM, Geoff. Doing interpretive research. European journal of information systems, v. 15, n. 3, p. 320-330, 2006. [GS Search]

WICKS, Andrew C.; FREEMAN, R. Edward. Organization studies and the new pragmatism: Positivism, anti-positivism, and the search for ethics. Organization science, v. 9, n. 2, p. 123-140, 1998. [GS Search] 